\title{
Fundamental Properties and Optimal Gains of a Steady-State Velocity Measured $\alpha-\beta$ Tracking Filter
}

\author{
Kenshi Saho \\ Department of Electronic and Computer Engineering, Ritsumeikan University, Kusatsu, Japan \\ Email: saho@fc.ritsumei.ac.jp
}

Received 1 April 2014; revised 30 April 2014; accepted 15 May 2014

Copyright (C) 2014 by author and Scientific Research Publishing Inc. This work is licensed under the Creative Commons Attribution International License (CC BY). http://creativecommons.org/licenses/by/4.0/ c) (i) Open Access

\begin{abstract}
This paper clarifies the steady-state properties and performance of an $\alpha-\beta$ filter for moving target tracking using both position and velocity measurements. We call this filter velocity measured $\alpha-\beta$ $(\mathrm{VM}-\alpha-\beta)$ filter. We first derive the stability condition and steady-state predicted errors as fundamental properties of the VM- $\alpha-\beta$ filter. The optimal gains for representative motion models are then derived from the Kalman filter equations. Theoretical and numerical analyses verify that VM- $\alpha-\beta$ filters with these optimal gains realize more accurate tracking than conventional $\alpha-\beta$ filters when the filter gains are relatively large. Our study reveals the conditions under which the predicted errors of the VM- $\alpha-\beta$ filters are less than those of conventional $\alpha-\beta$ filters. Moreover, numerical simulations clarify that the variance of the tracking error of the VM- $\alpha-\beta$ filters is approximately $3 / 4$ of that of the conventional $\alpha-\beta$ filters in realistic situations, even when the accuracy of the position/velocity measurements is the same.
\end{abstract}

\section{Keywords}

$\alpha-\beta$ Filter, Moving Target Tracking, Velocity Measurement, Kalman Filter, Optimal Gain

\section{Introduction}

Various monitoring systems that use remote sensors require the capability to accurately track moving targets. For such applications, representative approaches are to use a Kalman filter and an $\alpha-\beta(-\gamma)$ filter [1]. Kalman filters track moving targets based on a linear mechanical model of the target's motion [1] [2]. The use of improved algorithms in Kalman filters, such as the extended Kalman filter and particle filter, enables targets with complicated motions to be tracked by various organizations, such as air traffic control and intelligent transport systems 
[3]-[5]. However, the computational load of the Kalman and its extended filters is relatively large, and there are some cases where the use of these filters is impractical. Moreover, it is difficult to estimate their tracking performance analytically, and so the design of these filters is conducted empirically [1].

An $\alpha-\beta$ filter is a simple and effective tracking filter that assumes constant velocity during the sampling interval [1] [6]-[12]. Because of its small computational load, the use of $\alpha-\beta$ filters has been proposed in various tracking systems. Moreover, the $\alpha-\beta$ filter is also useful for the design of tracking systems using other filters (Kalman filter, etc.), because its tracking performance can be analytically predicted and a steady-state $\alpha-\beta$ filter can be obtained as a steady-state Kalman filter [6] [11]. Thus, many researchers have reported the properties, performance, and optimal gain settings of the $\alpha-\beta$ filter. Benedict and Bordner [7] derived an optimal gain relation for a random velocity target, and Kalata [10] found a similar relation for a random acceleration model while defining an effective design parameter known as the tracking index. These studies derived optimal gains in the steady state from the Kalman filter equations for simple and practical motion models. Based on these fundamental works, effective gain-setting algorithms for various maneuvering targets have been widely investigated [13]-[16].

The majority of conventional tracking filters measure only the position of their target. This is because they are mainly adapted for radar or global positioning systems that acquire only position information. However, thanks to technical advances in recent years, monitoring systems using many radars and/or networks composed of various sensors (e.g., cameras, lasers, ultrasound sensors, and acceleration sensors) have been realized [17]. These systems can measure the position, velocity, and acceleration of a target, and we would expect an improvement in tracking performance by using these measured parameters. For design of such tracking systems, the $\alpha-\beta$ filter using measurement of position, velocity, and acceleration should be effective. However, although some studies on $\alpha-\beta$ filter [18] [19], steady-state Kalman filter [20], and parameter tuning of Kalman filter [21]-[24] had been conducted for tracking systems measuring velocity, these systems had been designed empirically for each application. Thus, there are no reports on the general theory of the fundamental properties and performance of an $\alpha-\beta$ filter that uses parameters other than position. Therefore, the appropriate design of tracking filters that measure parameters such as velocity is difficult at present.

This paper focuses on the fundamental steady-state properties of an $\alpha-\beta$ filter that measures both position and velocity. From the Kalman filter equations, fundamental properties of a velocity measured $\alpha-\beta(\mathrm{VM}-\alpha-\beta)$ filter and optimal gains are analytically derived. Based on the derived properties, we clarify the performance of the VM- $\alpha-\beta$ filter, and compare it with that of conventional $\alpha-\beta$ filters. Moreover, numerical simulations clarify that the VM- $\alpha-\beta$ filter with sufficiently accurate velocity measurements realizes accurate tracking in a realistic situation, even when the performance of the conventional $\alpha-\beta$ filter deteriorates.

\section{System Model and Tracking Filter Basics}

This section first explains the target and measurement model assumed in this paper. The Kalman filter equations and fundamental properties of the conventional $\alpha-\beta$ filter are then described. The new analysis examined in this paper is based on these conventional filter basics.

\subsection{Target/Measurement Model and Kalman Filter Equations}

A tracking filter predicts the state of a moving target using measured data based on a dynamical model. In general, the state is composed of the position and velocity, and sensors measure the position of the target. The typical target dynamical model is [1]

$$
\boldsymbol{x}_{t}(k)=\boldsymbol{\Phi}(k-1) \boldsymbol{x}_{t}(k-1)+\Gamma_{1}(k-1) \boldsymbol{w}(k-1)
$$

where $\boldsymbol{x}_{t}(k)$ is the true target state vector at time $k T, T$ is the sampling interval, $\boldsymbol{\Phi}(k)$ is a transition matrix from $k T$ to $(k+1) T, \boldsymbol{w}(k)$ is a white Gaussian process noise with zero mean and covariance $\boldsymbol{Q}(k)$, and $\boldsymbol{\Gamma}_{1}(k)$ is a matrix that specifies the effect of $w(k)$. The measurement from a target is modeled as [1]

$$
\mathbf{z}(k)=\boldsymbol{H} \boldsymbol{x}_{t}(k)+\Gamma_{2}(k) \boldsymbol{v}(k)
$$

where $\mathbf{z}(k)$ is the measurement vector, $\boldsymbol{H}$ is a measurement matrix, $\boldsymbol{v}(k)$ is white Gaussian measurement noise with zero mean and covariance matrix $\boldsymbol{B}(k)$, and $\boldsymbol{\Gamma}_{2}(k)$ is a matrix that specifies the effect of $\boldsymbol{v}(k)$. The tracking system estimates the state vector based on these equations. 
The Kalman filters equentially estimates state vectors from the following equations [2]:

$$
\begin{gathered}
\overline{\boldsymbol{x}}(k)=\boldsymbol{\Phi}(k-1) \hat{\boldsymbol{x}}(k-1) \\
\overline{\boldsymbol{P}}(k)=\boldsymbol{\Phi}(k-1) \hat{\boldsymbol{P}}(k-1) \boldsymbol{\Phi}^{\mathrm{T}}(k-1)+\Gamma_{1}(k-1) \boldsymbol{Q}(k-1) \Gamma_{1}^{\mathrm{T}}(k-1) \\
\boldsymbol{K}(k)=\overline{\boldsymbol{P}}(k) \boldsymbol{H}^{\mathrm{T}}\left[\boldsymbol{H} \overline{\boldsymbol{P}}(k) \boldsymbol{H}^{\mathrm{T}}+\boldsymbol{\Gamma}_{2}(k) \boldsymbol{B}(k) \Gamma_{2}^{\mathrm{T}}(k)\right]^{-1} \\
\hat{\boldsymbol{x}}(k)=\overline{\boldsymbol{x}}(k)+\boldsymbol{K}(k)[\mathbf{z}(k)-\boldsymbol{H} \overline{\boldsymbol{x}}(k)] \\
\hat{\boldsymbol{P}}(k)=\overline{\boldsymbol{P}}(k)-\boldsymbol{K}(k) \boldsymbol{H} \overline{\boldsymbol{P}}(k)
\end{gathered}
$$

where forecasts and estimates are denoted by ${ }^{-}$and ${ }^{\wedge}$, respectively, ${ }^{\mathrm{T}}$ denotes the transpose, $\boldsymbol{P}(k)$ is a covariance matrix of errors, and $\boldsymbol{K}(k)$ is the Kalman gain. The Kalman filter estimates the optimal state vector whose predicted error is the minimum in the assumed target model.

\subsection{Conventional $\alpha-\beta$ Filter and Its Steady-State Performance}

The $\alpha-\beta$ filter estimates the position and velocity of a moving target using a position measured by sensors, and is defined by the following equations [7]:

$$
\begin{gathered}
x_{\mathrm{s}}(k)=x_{\mathrm{p}}(k)+\alpha\left[x_{\mathrm{o}}(k)-x_{\mathrm{p}}(k)\right] \\
v_{\mathrm{s}}(k)=v_{\mathrm{p}}(k)+(\beta / T)\left[x_{\mathrm{o}}(k)-x_{\mathrm{p}}(k)\right] \\
x_{\mathrm{p}}(k)=x_{\mathrm{s}}(k-1)+T v_{\mathrm{s}}(k-1) \\
v_{\mathrm{p}}(k)=v_{\mathrm{s}}(k-1)
\end{gathered}
$$

where $x_{\mathrm{s}}(k)$ is the smoothed target position at time $k T, x_{0}(k)$ is the measured target position, $x_{\mathrm{p}}(k)$ is the predicted target position, $v_{\mathrm{s}}(k)$ is the smoothed target velocity, $v_{\mathrm{p}}(k)$ is the predicted target velocity, and $\alpha$ and $\beta$ are filter gains. Equations (8)-(11) are obtained from the Kalman filter Equations (3)-(7) using a constant velocity model [7].

To evaluate the performance of $\alpha-\beta$ filter tracking, the following two steady-state errors can be derived from Equations (8)-(11).

1) Steady-state error for linear uniform motion (smoothing performance): an important function of the tracking filter is the reduction of random errors caused by noise. One indicator of this performance is the steady-state error of the target with linear uniform motion considering sensor noise. We assume the variance of the noise of $x_{0}(k)$ is $B_{\mathrm{x}}$, and that the target moves with constant velocity. The variance of the predicted target position in the steady state is expressed as [6] [9]

$$
\sigma_{\mathrm{p}}^{2}=\mathrm{E}\left[\left(x_{\mathrm{p}}(k)-x_{\mathrm{t}}(k)\right)^{2}\right]=\frac{2 \alpha^{2}+2 \beta+\alpha \beta}{\alpha(4-2 \alpha-\beta)} B_{\mathrm{x}}
$$

where $\mathrm{E}\left[\mathrm{]}\right.$ indicates the mean. This paper calls $\sigma_{\mathrm{p}}^{2}$ the smoothing performance.

2) Steady-state error for constant acceleration target (tracking performance): the filter is required to track an accelerating target. The tracking accuracy for a target with acceleration is evaluated by the steady-state bias error for a target under constant acceleration. When $x_{0}(k)=1 / 2 a_{\mathrm{c}}(k T)^{2}\left(a_{\mathrm{c}}\right.$ is the constant acceleration), the steadystate predicted error is expressed as [6] [9]

$$
e_{\text {fin }}=\lim _{k \rightarrow \infty}\left[x_{\mathrm{o}}(k)-x_{\mathrm{p}}(k)\right]=a_{\mathrm{c}} T^{2} / \beta
$$

This paper calls $e_{\text {fin }}$ the tracking performance.

\subsection{Optimal Gains of an $\alpha-\beta$ Filter}

The optimal gains of the $\alpha-\beta$ filter minimize the steady-state errors, and are derived using the Kalman filter equations [7]-[10]. The following two optimal gain relations for representative motion models are known: 
1) Random velocity process noise model [7] [8]: let $\boldsymbol{w}(k)$ of Equation (1) be the first derivative of the target position:

$$
\begin{gathered}
\Gamma_{1}(k)=\left(\begin{array}{ll}
T & 1
\end{array}\right)^{\mathrm{T}} \\
\Gamma_{1}(k) Q(k) \Gamma_{1}^{\mathrm{T}}(k)=\left(\begin{array}{cc}
T^{2} & T \\
T & 1
\end{array}\right) Q(k)
\end{gathered}
$$

For this model, the optimal relationship between the gains is known to be [7]

$$
\beta=\alpha^{2} /(2-\alpha)
$$

Equation (14) is the so-called Benedict-Bordner relation (BBR) [12], and this paper calls an $\alpha-\beta$ filter having this relation a BBR filter. This filter is stable when $0<\alpha \leq 1$.

2) Random acceleration process noise model [8] [10]: let $\boldsymbol{w}(k)$ be the second derivative of the target position:

$$
\begin{gathered}
\Gamma_{1}(k)=\left(\begin{array}{ll}
T^{2} / 2 & T
\end{array}\right)^{\mathrm{T}} \\
\Gamma_{1}(k) Q(k) \Gamma_{1}^{\mathrm{T}}(k)=\left(\begin{array}{cc}
T^{4} / 4 & T^{3} / 2 \\
T^{3} / 2 & T^{2}
\end{array}\right) Q(k)
\end{gathered}
$$

For this model, the optimal relationship between the gains is known to be [10]

$$
\beta=2(2-\alpha)-4 \sqrt{1-\alpha}
$$

Equation (17) is known as the Kalata relation (KR) [12], and this paper calls an $\alpha-\beta$ filter having this relation a KR filter. This filter is stable when $0<\alpha<1$.

The BBR and KR filters are basic tracking filters for realizing the desired performance in the steady state. Consequently, this paper compares their performance with that of the VM- $\alpha-\beta$ filter defined in the next section.

\section{Velocity Measured $\alpha-\beta$ Filter and Its Fundamental Properties}

This section firstly defines an $\alpha-\beta$ filter using measurements of both position and velocity we deal with in this paper. Then, we derive its stability condition and filter performance indicators as fundamental steady-state properties.

\subsection{Definition}

As for the conventional $\alpha-\beta$ filter, an $\alpha-\beta$ filter using measured velocity $v_{0}(k)$ can be defined with the Kalman filter equations. We substitute the following into Equations (3)-(7):

$$
\begin{gathered}
\overline{\boldsymbol{x}}(k)=\left(\begin{array}{ll}
x_{\mathrm{p}}(k) & v_{\mathrm{p}}(k)
\end{array}\right)^{\mathrm{T}} \\
\hat{\boldsymbol{x}}(k)=\left(\begin{array}{ll}
x_{\mathrm{s}}(k) & v_{\mathrm{s}}(k)
\end{array}\right)^{\mathrm{T}} \\
\boldsymbol{\Phi}(k)=\left(\begin{array}{cc}
1 & T \\
0 & 1
\end{array}\right) \\
\boldsymbol{z}(k)=\left(\begin{array}{ll}
x_{\mathrm{o}}(k) & v_{\mathrm{o}}(k)
\end{array}\right)^{\mathrm{T}} \\
\boldsymbol{H}(k)=\left(\begin{array}{ll}
1 & 0 \\
0 & 1
\end{array}\right) \\
\boldsymbol{K}(k)=\left(\begin{array}{cc}
\alpha & 0 \\
0 & \beta
\end{array}\right)
\end{gathered}
$$

This paper assumes that Kalman gains other than the main diagonal elements are zero, as in Equation (25), to avoid increasing the number of design parameters. As indicated in Equations (5) and (7), this assumption is equivalent to neglecting the covariance of the errors in position and velocity. As a result of this substitution, 
Equations (8), (10), and (11) are same as the conventional $\alpha-\beta$ filter and Equation (9) becomes

$$
v_{\mathrm{s}}(k)=v_{\mathrm{p}}(k)+\beta\left[v_{\mathrm{o}}(k)-v_{\mathrm{p}}(k)\right]
$$

We call the tracking filter defined by Equations (8), (10), (11), and (26) the VM- $\alpha-\beta$ filter.

\subsection{Stability Condition}

To clarify the stability condition of the VM- $\alpha-\beta$ filter, we examine the relationship between the input and output signals in z-space. Applying a z-transform to Equations (8), (10), (11), and (26), the relationships between input signals $x_{0}(k)$ and $v_{0}(k)$ and output signals $x_{\mathrm{p}}(k)$ and $v_{\mathrm{p}}(k)$ in $z$-space can be written as:

$$
\begin{aligned}
X_{\mathrm{p}}(z)= & \frac{\alpha}{z+\alpha-1} X_{\mathrm{o}}(z)+\frac{\beta z}{(z+\alpha-1)(z+\beta-1)} T V_{\mathrm{o}}(z) \\
& V_{\mathrm{p}}(\mathrm{z})=\beta V_{\mathrm{o}}(\mathrm{z}) /(\mathrm{z}+\beta-1)
\end{aligned}
$$

Thus, the stability condition of the VM- $\alpha-\beta$ filter is easily derived from the characteristic polynomial of $(z+\alpha-1)(z+\beta-1)$, resulting in the gains constraints:

$$
0<\alpha<2 \text { and } 0<\beta<2
$$

This condition means that the VM- $\alpha-\beta$ filter is stable for practical use, because gains are generally in the range $0<\alpha<1$ and $0<\beta<1$ to obtain a smoothing effect in the filter.

\subsection{Tracking Performance}

We can derive the predicted errors in the steady state to evaluate the tracking/smoothing performance. First, the steady-state error for a target with constant acceleration $a_{\mathrm{c}}$ is derived to evaluate the tracking performance. The result of the derivation is

$$
e_{\text {fin }}=\lim _{k \rightarrow \infty}\left[x_{\mathrm{o}}(k)-x_{\mathrm{p}}(k)\right]=(2-\beta) a_{\mathrm{c}} T^{2} /(2 \alpha \beta)
$$

The derivation of Equation (30) is as follows. From Equation (27), the z-transform of $e_{\mathrm{p}}(k)=x_{0}(k)-x_{\mathrm{p}}(k)$ is given by

$$
E_{\mathrm{p}}(z)=X_{\mathrm{o}}(z)-X_{\mathrm{p}}(z)=\frac{z-1}{z+\alpha-1} X_{\mathrm{o}}(z)-\frac{\beta z}{(z+\alpha-1)(z+\beta-1)} V_{\mathrm{o}}(z)
$$

Here, because we assume the target has constant acceleration $a_{\mathrm{c}}$, the measured position and velocity are given as:

$$
\begin{gathered}
x_{\mathrm{o}}(k)=a_{\mathrm{c}}(k T)^{2} / 2 \\
v_{\mathrm{o}}(k)=a_{\mathrm{c}} k T
\end{gathered}
$$

and their $z$-transforms are:

$$
\begin{array}{r}
X_{\mathrm{o}}(z)=z(z+1) a_{\mathrm{c}} T^{2} /\left[2(z-1)^{3}\right] \\
V_{\mathrm{o}}(z)=z a_{\mathrm{c}} T /(z-1)^{2}
\end{array}
$$

Substituting these into Equation (31), we obtain

$$
E_{\mathrm{p}}(z)=\frac{z(z+1)(z+\beta-1)-2 \beta z}{2(z-1)^{2}(z+\alpha-1)(z+\beta-1)} a_{\mathrm{c}} T^{2}
$$

Using Equation (36), the final value theorem, and l'Hôpital's rule, we have Equation (30).

\subsection{Smoothing Performance}

Next, we derive the smoothing performance. As mentioned in Section 3.1, we assume that the errors in position 
and velocity are uncorrelated. The covariance matrix of the measurement noise is thus expressed as

$$
\Gamma_{2}(k) \boldsymbol{B}(k) \Gamma_{2}^{\mathrm{T}}(k)=\left(\begin{array}{cc}
B_{\mathrm{x}} & 0 \\
0 & B_{\mathrm{v}}
\end{array}\right)
$$

where $B_{\mathrm{v}}$ is the variance of the measurement noise of $v_{\mathrm{o}}(k)$. Under the assumption of Equation (37), the variance in steady-state errors of the predicted position for a constant velocity target is derived as

$$
\sigma_{\mathrm{p}}^{2}=\mathrm{E}\left[x_{\mathrm{p}}(k)-x_{\mathrm{t}}(k)\right]=\frac{\alpha}{2-\alpha} B_{\mathrm{x}}+\frac{\beta}{\alpha(2-\alpha)(2-\beta)} T^{2} B_{\mathrm{v}}
$$

The derivation of Equation (38) is as follows. First, we determine the variance of the smoothed position and velocity errors. From Equations (8), (10), (11), and (26), the errors in the smoothed position and velocity are:

$$
\begin{gathered}
x_{\mathrm{s}}(k)-x_{\mathrm{t}}(k)=(1-\alpha)\left\{\left(x_{\mathrm{s}}(k-1)-x_{\mathrm{t}}(k-1)\right)+T\left(v_{\mathrm{s}}(k-1)-v_{\mathrm{t}}(k-1)\right)\right\}+\alpha\left(x_{\mathrm{o}}(k)-x_{\mathrm{t}}(k)\right) \\
v_{\mathrm{s}}(k)-v_{\mathrm{t}}(k)=(1-\beta)\left(v_{\mathrm{s}}(k-1)-v_{\mathrm{t}}(k-1)\right)+\beta\left(v_{\mathrm{o}}(k)-v_{\mathrm{t}}(k)\right)
\end{gathered}
$$

Therefore, the variances of these errors are calculated as

$$
\begin{aligned}
& \mathrm{E}\left[\left(x_{\mathrm{s}}(k)-x_{\mathrm{t}}(k)\right)^{2}\right] \\
& =(1-\alpha)^{2}\left\{\mathrm{E}\left[\left(x_{\mathrm{s}}(k-1)-x_{\mathrm{t}}(k-1)\right)^{2}\right]+2 T \mathrm{E}\left[\left(x_{\mathrm{s}}(k-1)-x_{\mathrm{t}}(k-1)\right)\left(v_{\mathrm{s}}(k-1)-v_{\mathrm{t}}(k-1)\right)\right]\right. \\
& \left.+T^{2} \mathrm{E}\left[\left(v_{\mathrm{s}}(k-1)-v_{\mathrm{t}}(k-1)\right)^{2}\right]\right\}+2 \alpha(1-\alpha)\left\{\mathrm{E}\left[\left(x_{\mathrm{s}}(k-1)-x_{\mathrm{t}}(k-1)\right)\left(x_{\mathrm{o}}(k)-x_{\mathrm{t}}(k)\right)\right]\right. \\
& \left.+T \mathrm{E}\left[\left(x_{\mathrm{o}}(k)-x_{\mathrm{t}}(k)\right)\left(v_{\mathrm{s}}(k-1)-v_{\mathrm{t}}(k-1)\right)\right]\right\}+\alpha^{2} \mathrm{E}\left[\left(x_{\mathrm{o}}(k)-x_{\mathrm{t}}(k)\right)^{2}\right] \\
& \mathrm{E}\left[\left(v_{\mathrm{s}}(k)-v_{\mathrm{t}}(k)\right)^{2}\right]= \\
& +1-\beta)^{2} \mathrm{E}\left[\left(v_{\mathrm{s}}(k-1)-v_{\mathrm{t}}(k-1)\right)^{2}\right] \\
& +2 \beta(1-\beta) \mathrm{E}\left[\left(v_{\mathrm{s}}(k-1)-v_{\mathrm{t}}(k-1)\right)\left(v_{\mathrm{o}}(k)-v_{\mathrm{t}}(k)\right)\right] \\
& +\beta^{2} \mathrm{E}\left[\left(v_{\mathrm{o}}(k)-v_{\mathrm{t}}(k)\right)^{2}\right]
\end{aligned}
$$

Since the position and velocity errors are uncorrelated and we are considering the steady state, we can assume:

$$
\begin{gathered}
\mathrm{E}\left[\left(x_{\mathrm{s}}(k)-x_{\mathrm{t}}(k)\right)^{2}\right]=\mathrm{E}\left[\left(x_{\mathrm{s}}(k-1)-x_{\mathrm{t}}(k-1)\right)^{2}\right]=\sigma_{\mathrm{xs}}^{2} \\
\mathrm{E}\left[\left(v_{\mathrm{s}}(k)-v_{\mathrm{t}}(k)\right)^{2}\right]=\mathrm{E}\left[\left(v_{\mathrm{s}}(k-1)-v_{\mathrm{t}}(k-1)\right)^{2}\right]=\sigma_{\mathrm{vs}}^{2} \\
\mathrm{E}\left[\left(x_{\mathrm{s}}(k-1)-x_{\mathrm{t}}(k-1)\right)\left(v_{\mathrm{s}}(k-1)-v_{\mathrm{t}}(k-1)\right)\right]=0 \\
\mathrm{E}\left[\left(x_{\mathrm{o}}(k)-x_{\mathrm{t}}(k)\right)^{2}\right]=B_{\mathrm{x}} \\
\mathrm{E}\left[\left(v_{\mathrm{o}}(k)-v_{\mathrm{t}}(k)\right)^{2}\right]=B_{\mathrm{v}} \\
\mathrm{E}\left[\left(x_{\mathrm{o}}(k)-x_{\mathrm{t}}(k)\right)\left(v_{\mathrm{s}}(k-1)-v_{\mathrm{t}}(k-1)\right)\right]=0
\end{gathered}
$$

Because the smoothed parameters are a linear combination of the measured parameters, the following relations are satisfied:

$$
\begin{aligned}
& \mathrm{E}\left[\left(x_{\mathrm{s}}(k-1)-x_{\mathrm{t}}(k-1)\right)\left(x_{\mathrm{o}}(k)-x_{\mathrm{t}}(k)\right)\right]=0 \\
& \mathrm{E}\left[\left(v_{\mathrm{s}}(k-1)-v_{\mathrm{t}}(k-1)\right)\left(v_{\mathrm{o}}(k)-v_{\mathrm{t}}(k)\right)\right]=0
\end{aligned}
$$


Substituting Equations (43)-(50) into Equations (41) and (42), the variances are given by:

$$
\begin{gathered}
\sigma_{\mathrm{vs}}^{2}=\beta B_{\mathrm{v}} /(2-\beta) \\
\sigma_{\mathrm{xs}}^{2}=\frac{\alpha}{2-\alpha} B_{\mathrm{x}}+\frac{\beta(1-\alpha)^{2}}{\alpha(2-\alpha)(2-\beta)} T^{2} B_{\mathrm{v}}
\end{gathered}
$$

Using these variances, we can derive the variance of the predicted error. From Equations (10) and (43)-(45), the variance of the predicted error is expressed as

$$
\begin{aligned}
\sigma_{\mathrm{p}}^{2} & =\mathrm{E}\left[\left(x_{\mathrm{p}}(k)-x_{\mathrm{t}}(k)\right)^{2}\right]=\mathrm{E}\left[\left(x_{\mathrm{s}}(k-1)-x_{\mathrm{t}}(k-1)\right)^{2}\right] \\
& +2 T \mathrm{E}\left[\left(x_{\mathrm{s}}(k-1)-x_{\mathrm{t}}(k-1)\right)\left(v_{\mathrm{s}}(k-1)-v_{\mathrm{t}}(k-1)\right)\right] \\
& +T^{2} \mathrm{E}\left[\left(v_{\mathrm{s}}(k-1)-v_{\mathrm{t}}(k-1)\right)^{2}\right] \\
& =\sigma_{\mathrm{xs}}^{2}+T^{2} \sigma_{\mathrm{vs}}^{2}
\end{aligned}
$$

Substituting Equations (51) and (52) into Equation (53), we have Equation (38).

\section{Optimal Gains of the VM- $\alpha-\beta$ Filter}

Using the tracking/smoothing performance given by Equations (30) and (38), we can evaluate the performance of the VM- $\alpha-\beta$ filter. However, as shown in these equations, there is a trade-off between these steady-state errors. For example, although a relatively small $\alpha$ is appropriate to reduce $\sigma_{\mathrm{p}}^{2}$ in Equation (38) when $B_{\mathrm{x}}$ is large compared with $T^{2} B_{\mathrm{v}}$, a small value of $\alpha$ serves to increase the value of $e_{\text {fin }}$ in Equation (30). To realize better performance while considering this trade-off, setting the optimal gains derived from the Kalman filter has been shown to be an effective approach [6]-[10]. This section derives optimal gain relationships for the VM- $\alpha-\beta$ filter from the Kalman filter equations for the two representative motion models described in Section 2.3.

\subsection{Optimal Gain Relationship for Random Velocity Model}

In this subsection, we consider the optimal gains when the process noise is a zero-mean random velocity, as given by Equations (14) and (15), such as in the BBR filter. For this model, the optimal gain relationship is given by

$$
\beta=\frac{\alpha^{2}}{\alpha^{2}+R_{\mathrm{B}}(1-\alpha)}
$$

where

$$
R_{\mathrm{B}}=T^{2} B_{\mathrm{v}} / B_{\mathrm{x}}
$$

We call the VM- $\alpha-\beta$ filter whose gains satisfy Equation (54) the random-velocity velocity-measured (RVVM) filter.

The derivation of Equation (54) is as follows. Optimal gains are derived from the relationship between the gain matrix $\boldsymbol{K}(k)$ and variance matrix $\boldsymbol{P}(k)$ of the Kalman filter equations. As we have assumed that the covariance of the position and velocity is zero, let the covariance matrices be:

$$
\begin{aligned}
& \overline{\mathbf{P}}(k)=\left(\begin{array}{cc}
\bar{P}^{11}(k) & 0 \\
0 & \bar{P}^{22}(k)
\end{array}\right) \\
& \hat{\boldsymbol{P}}(k)=\left(\begin{array}{cc}
\hat{P}^{11}(k) & 0 \\
0 & \hat{P}^{22}(k)
\end{array}\right)
\end{aligned}
$$

Substituting Equations (15), (22), (56), and (57) into Equation (4), we obtain:

$$
\bar{P}^{11}(k)=\hat{P}^{11}(k-1)+T^{2} \hat{P}^{22}(k-1)+T^{2} Q(k-1)
$$




$$
\bar{P}^{22}(k)=\hat{P}^{22}(k-1)+Q(k-1)
$$

Substituting Equations (25), (56), and (57) into Equation (7), we obtain:

$$
\begin{aligned}
& \hat{P}^{11}(k)=(1-\alpha) \bar{P}^{11}(k-1) \\
& \hat{P}^{22}(k)=(1-\beta) \bar{P}^{22}(k-1)
\end{aligned}
$$

Equation (5) can be rewritten as [25]

$$
\boldsymbol{K}(k)=\hat{\boldsymbol{P}}(k) \boldsymbol{H}^{\mathrm{T}}\left[\boldsymbol{\Gamma}_{2}(k) \boldsymbol{B}(k) \boldsymbol{\Gamma}_{2}^{\mathrm{T}}(k)\right]^{-1}
$$

Substituting Equations (24), (37), (60), (61) into (62), the gains can be expressed as:

$$
\begin{aligned}
& \alpha=\bar{P}^{11}(k) /\left(\bar{P}^{11}(k)+B_{\mathrm{x}}\right) \\
& \beta=\bar{P}^{22}(k) /\left(\bar{P}^{22}(k)+B_{\mathrm{v}}\right)
\end{aligned}
$$

From Equations (58), (60), (61), and (63), and assuming the steady state (i.e. all variables do not depend on $k$ ), we obtain

$$
\alpha=\frac{\alpha B_{\mathrm{x}}+T^{2} \beta B_{\mathrm{v}}+T^{2} Q}{\alpha B_{\mathrm{x}}+T^{2} \beta B_{\mathrm{v}}+T^{2} Q+B_{\mathrm{x}}}
$$

Similarly, from Equations (59), (61), and (64), we obtain

$$
\beta=\frac{\beta B_{\mathrm{v}}+Q}{\beta B_{\mathrm{v}}+Q+B_{\mathrm{v}}}
$$

Simplifying Equations (65) and (66), we arrive at Equation (54).

Figure 1(a) shows the relationship between the gains $\alpha(0<\alpha \leq 1)$ and $\beta$ of the RV-VM filter for various $R_{\mathrm{B}}$ calculated with Equation (54), and that of the conventional BBR filter calculated with Equation (16). As shown in this figure, the gain of $\beta$ becomes large compared with the BBR filter when $R_{\mathrm{B}}$ is relatively small. When $R_{\mathrm{B}}$ is small, the reliability of the measured velocity is high. Consequently, the velocity gain $\beta$ becomes large to make best use of the measured velocity. In contrast, the conventional BBR filter estimates the velocity as the derivative of position, and the gain $\beta$ therefore becomes small to avoid instabilities in the estimation. In addition, when $R_{\mathrm{B}}$ is relatively large, the value of $\beta$ in the RV-VM filter becomes small compared with that in the BBR filter, because the measured velocity is unreliable.

\subsection{Optimal Gain Relationship for Random Acceleration Model}

This subsection considers the random acceleration process noise modeled by Equations (17) and (18), as in the KR filter. Using the same procedure as for the RV-VM filter in Equations (56)-(64) with Equation (18), we ob-

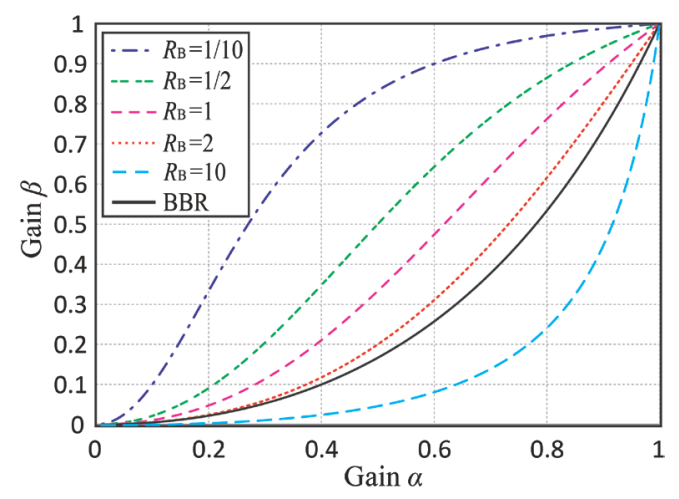

(a)

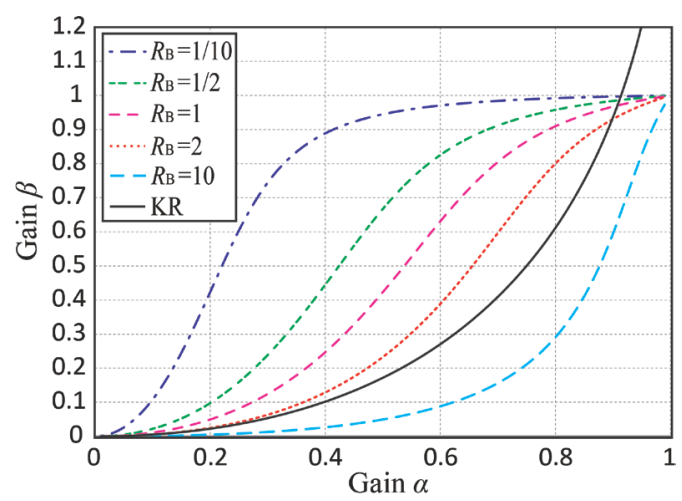

(b)

Figure 1. Relationship between optimal gains. (a) RV-VM and BBR filters; (b) RA-VM and KR filters. 
tain the steady-state gains corresponding to Equations (65) and (66) for this motion model as:

$$
\begin{gathered}
\alpha=\frac{\alpha B_{\mathrm{x}}+T^{2} \beta B_{\mathrm{v}}+T^{4} Q / 4}{\alpha B_{\mathrm{x}}+T^{2} \beta B_{\mathrm{v}}+T^{4} Q / 4+B_{\mathrm{x}}} \\
\beta=\frac{\beta B_{\mathrm{v}}+T^{2} Q}{\beta B_{\mathrm{v}}+T^{2} Q+B_{\mathrm{v}}}
\end{gathered}
$$

Thus, optimal gains relationship can be written using Equation (55) as

$$
\beta=2 \frac{\alpha^{2}+R_{\mathrm{B}}(1-\alpha)-\sqrt{(1-\alpha)^{2} R_{\mathrm{B}}^{2}-\alpha^{2}(1-\alpha) R_{\mathrm{B}}+\alpha^{4}}}{3 R_{\mathrm{B}}(1-\alpha)}
$$

We call the VM- $\alpha-\beta$ filter whose gains satisfy Equation (69) the random-acceleration velocity-measured (RA$\mathrm{VM}$ ) filter. Note that this filter is unstable when $R_{\mathrm{B}}=0$ or $\alpha=1$, as can be seen from Equation (69).

Figure 1(b) shows the relationship between gains $\alpha(0<\alpha<1)$ and $\beta$ of the RA-VM filter for various $R_{\mathrm{B}}$ calculated with Equation (69), and that of the conventional KR filter calculated with Equation (19). Although the relationship is qualitatively similar to that of the RV-VM and BBR filters, $\beta$ increases faster than in the RV-VM and BBR filters. This is because the RA-VM and KR filters consider randomly varying acceleration, and the gains thus become relatively large to realize sufficient tracking performance, as indicated in Equations (13) and (30).

\section{Numerical Evaluation}

In this section, we use numerical examples to evaluate the tracking and smoothing performance of the derived VM- $\alpha-\beta$ filters (RV-VM and RA-VM filters), and compare these results with those from conventional $\alpha-\beta$ filters (BBR and KR filters). First, the filter performance is evaluated based on the derived steady-state predicted errors. Then, we simulate a maneuvering target tracking to show the performance in a realistic situation.

\subsection{Performance Evaluation of RV-VM Filter and Comparison with BBR Filter}

This subsection evaluates the filter performance of the RV-VM and BBR filters. These are derived from the same process noises (random velocity model). The gains, smoothing performance, and tracking performance of the RV-VM filter are calculated with Equations (54), (38), and (30), respectively. Those of the BBR filter are calculated with Equations (16), (12), and (13). We assume that the sampling interval $T$, the variance of the measured position error $B_{\mathrm{x}}$, and the acceleration $a_{\mathrm{c}}$ used in the tracking performance evaluation are normalized to 1 .

Figure 2 shows the smoothing performance of the RV-VM filter for various $R_{\mathrm{B}}$ and the results given by the BBR filter. When $\alpha$ is relatively large, the smoothing performance of the RV-VM filter with small $R_{\mathrm{B}}$ is better than that of the BBR filter. In particular, when $R_{\mathrm{B}}=1$ and 2, although the accuracy of the velocity sensor is the same or worse than that of the position sensor, the smoothing performance of the RV-VM filter is significantly better than that of the BBR filter when (approximately) $\alpha>0.7$. This indicates that the RV-VM filter enjoys im-

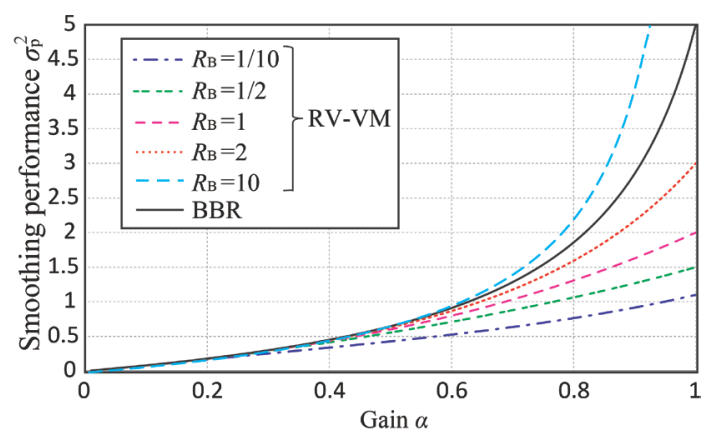

Figure 2. Smoothing performance of the RV-VM and BBR filters. 
proved performance using the measured velocity, even when the velocity sensor has relatively poor accuracy. However, as can be seen when $R_{\mathrm{B}}=10$, the predicted error becomes worse compared with the BBR filter when the noise variance of the velocity measurement is extremely large. Thus, we should not use the measured velocity in this case. Then, we clarify the conditions under which the smoothing performance of the RV-VM filter is better than that of the conventional BBR filter using Equations (12), (16), (38), and (54). Substituting Equation (16) into Equation (12), the smoothing performance of the BBR filter is expressed as

$$
\sigma_{\mathrm{p}(\mathrm{BBR})}^{2}=\frac{\alpha(6-\alpha)}{\alpha^{2}-8 \alpha+8} B_{\mathrm{x}}
$$

and substituting Equation (54) into (38), the smoothing performance of the RV-VM filter is

$$
\sigma_{\mathrm{p}(\mathrm{RV}-\mathrm{VM})}^{2}=\frac{\alpha\left(\alpha^{2}-2 R_{\mathrm{B}} \alpha+3 R_{\mathrm{B}}\right)}{(2-\alpha)\left(\alpha^{2}-2 R_{\mathrm{B}} \alpha+2 R_{\mathrm{B}}\right)} B_{\mathrm{x}}
$$

As shown in Equations (70) and (71), $\sigma_{\mathrm{p}}^{2}$ of the RV-VM and BBR filters become same value when $R_{\mathrm{B}}=4$. Thus, the smoothing performance of the RV-VM filter is better than that of the BBR filter for all $\alpha$ when $R_{\mathrm{B}}<4$.

Figure 3(a) shows the tracking performance of the RV-VM and BBR filters. The same tendency as for the smoothing performance can be seen for relatively large $\alpha$. However, for small $\alpha$, the performance of the RV-VM filter with relatively large $R_{\mathrm{B}}$ is worse than that of the BBR filter. This means that, from the viewpoint of tracking performance, the RV-VM filter is unsuitable when $\alpha$ is small and $R_{\mathrm{B}}$ is large. However, unlike the smoothing performance, with sufficiently small $R_{\mathrm{B}}$, the tracking performance is efficiently improved for small $\alpha$. Figure 3(b) shows the tracking performance threshold $R_{\mathrm{BT}-\mathrm{RV}}$, which is the value of $R_{\mathrm{B}}$ for which $e_{\mathrm{fin}}$ of the RV-VM and BBR filters takes the same value for each $\alpha$. This means that the tracking performance of the RV-VM filter is better than that of the BBR filter when $R_{\mathrm{B}}<R_{\mathrm{BT}-\mathrm{RV}}$, indicating the conditions under which the predicted bias error of the RV-VM filter is smaller than that of the conventional BBR filter. Even for $R_{\mathrm{B}}=1, e_{\mathrm{fin}}$ in the RV-VM filter is smaller than that in the BBR filter when $\alpha>0.423$ which is calculated with Equations (12), (13), (30), and (38). These investigations show that both the tracking and smoothing performance of the RV-VM filter is better than that of the BBR filter, even when $R_{\mathrm{B}} \geq 1$, for relatively large $\alpha$. Moreover, for relatively small $\alpha$, although the improvement in performance is not so significant, the tracking and smoothing performance of the $\mathrm{RV}-\mathrm{VM}$ filter is enhanced when $R_{\mathrm{B}}$ is sufficiently small.

\subsection{Performance Evaluation of RA-VM Filter and Comparison with KR Filter}

This subsection evaluates the performance of the RA-VM and KR filters. These are derived from the random acceleration model expressed in Equations (17) and (18). The gains, smoothing performance, and tracking performance of the RA-VM filter are calculated with Equations (69), (38), and (30), respectively. Those of the KR filter are calculated with Equations (19), (12), and (13). As in the previous section, we assume that $T, B_{\mathrm{x}}$, and $a_{\mathrm{c}}$ used are normalized to 1 .

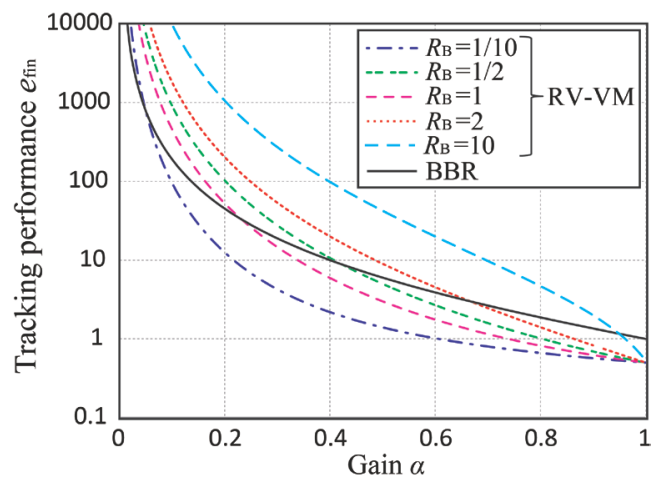

(a)

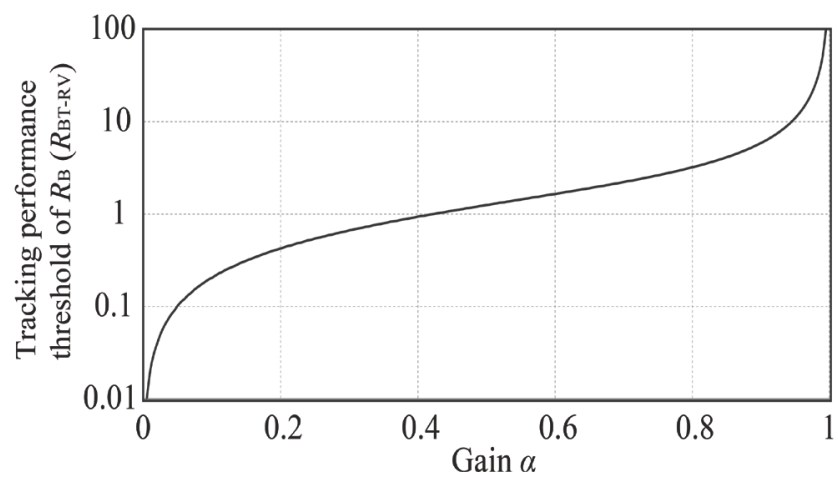

(b)

Figure 3. Tracking performance evaluation of the RV-VM and BBR filters. (a) Tracking performance $e_{\text {fin }}$ for each $\alpha$; (b) Tracking performance threshold $R_{\mathrm{BT}-\mathrm{RV}}$. 
Figure 4(a) shows the smoothing performance of the RA-VM filter with various $R_{\mathrm{B}}$ and the results given by the KR filter. The same tendency as for the RV-VM and BBR filters can be observed. When (approximately) $\alpha$ $>0.8$, the smoothing performance of the RA-VM filter is significantly better than that of the KR filter except for $R_{\mathrm{B}}=10$. Moreover, as shown in the results for $R_{\mathrm{B}}=10$, although the smoothing performance of the RA-VM filter is worse than that of the KR filter when (approximately) $\alpha>0.6$, the difference between them is slight. This suggests that the RA-VM filter is suitable for practical use in terms of smoothing performance, even when the velocity sensor reliability is low compared with that of the position sensor. Figure 4(b) shows the smoothing performance threshold $R_{\mathrm{BS}-\mathrm{RA}}$, which is the value of $R_{\mathrm{B}}$ for which $\sigma_{\mathrm{p}}{ }^{2}$ in the RA-VM and KR filters takes the same value for $0.4 \leq \alpha<1$. As indicated in this figure, the RA-VM filter with relatively large $\alpha$ realizes better accuracy. Even for $R_{\mathrm{B}} \geq 1$, the smoothing performance of the RA-VM filter is better than that of the KR filter in $\alpha>0.656$ which is calculated with Equations (12), (19), (38), and (69).

Figure 5 shows the tracking performance of the RA-VM and KR filters, and the tracking performance threshold $R_{\mathrm{BT}-\mathrm{RV}}$. This figure exhibits similar properties as for the RV-VM and BBR filters. Compared with the RV-VM and BBR filter results in Figure 3, the tracking performance of the RA-VM and KR filters is improved by the assumption of random acceleration.

\subsection{Comparison of RV-VM and RA-VM Filters}

We now compare the performance of the RV-VM and RA-VM filters. Figure 6(a) shows the difference in $\sigma_{\mathrm{p}}{ }^{2}$ given by the two filters. As shown in this figure, the values of $\sigma_{\mathrm{p}}^{2}$ of the RV-VM filter minus that of the RAVM filter are negative for all $\alpha$, which means that the smoothing performance of the RV-VM filter is better than that of the RA-VM filter. Analogously, Figure 6(b) shows the difference in the $e_{\mathrm{fin}}$ values given by the RV-VM

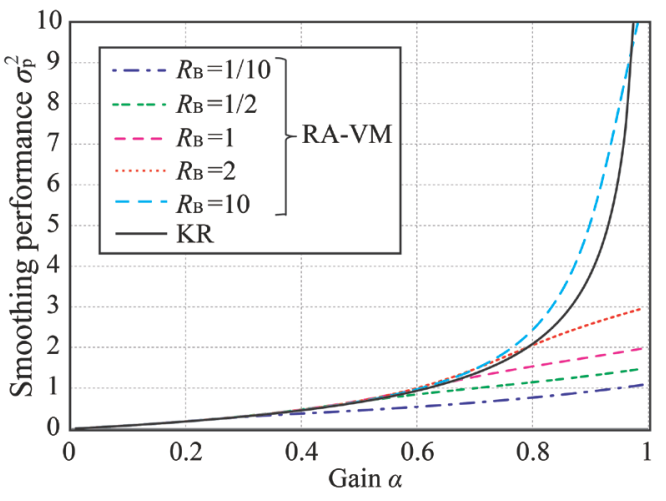

(a)

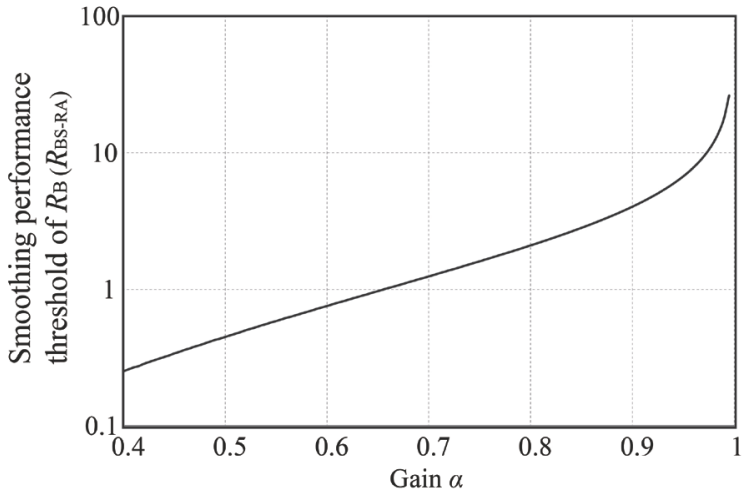

(b)

Figure 4. Smoothing performance evaluation of the RA-VM and KR filters. (a) Smoothing performance ${\sigma_{\mathrm{p}}}^{2}$ for each $\alpha$; (b) Smoothing performance threshold $R_{\mathrm{BS}-\mathrm{RA}}$.

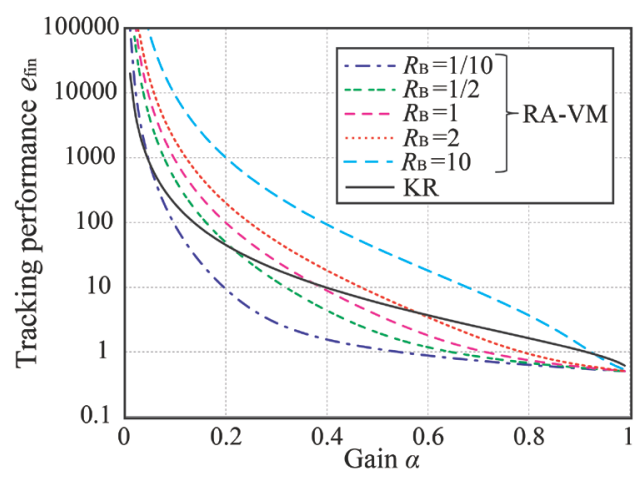

(a)

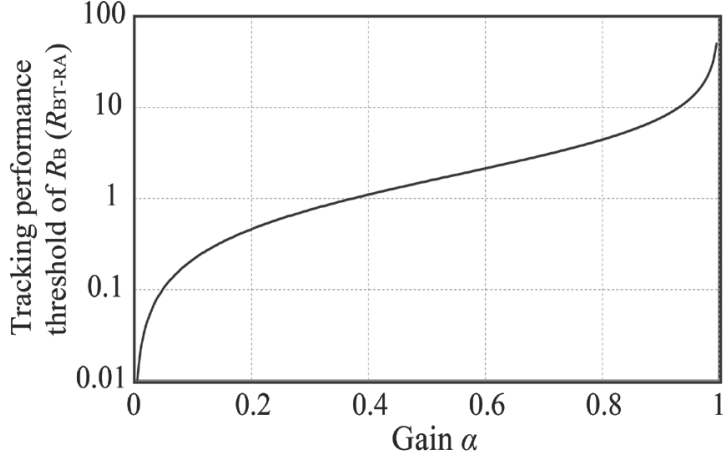

(b)

Figure 5. Tracking performance evaluation of the RA-VM and KR filters. (a) Tracking performance $e_{\text {fin }}$ for each $\alpha$; (b) Tracking performance threshold $R_{\mathrm{BT}-\mathrm{RA}}$. 


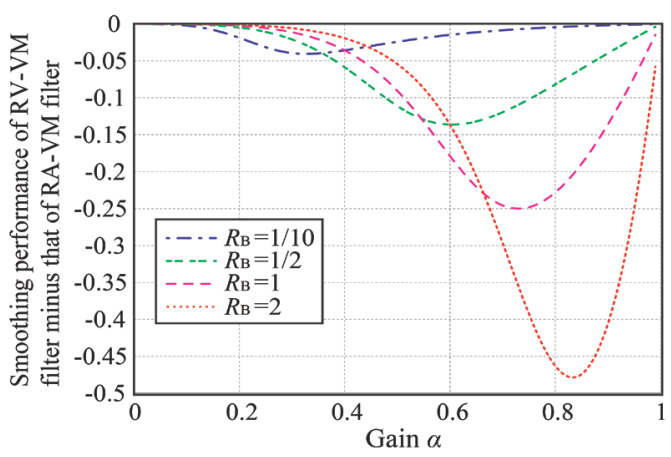

(a)

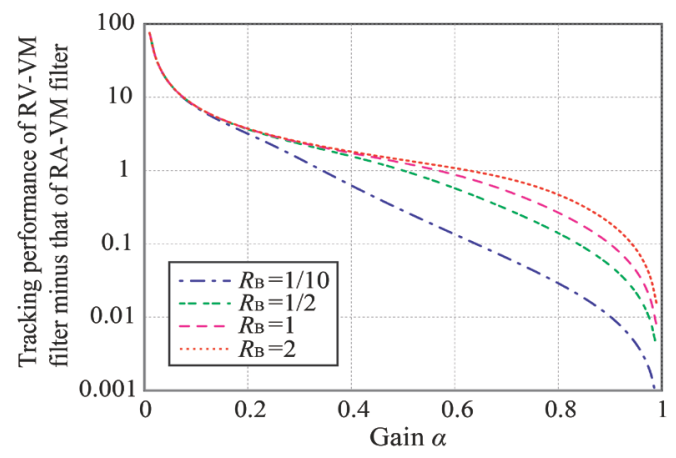

(b)

Figure 6. Performance difference between RV-VM and RA-VM filters. (a) Smoothing performance; (b) Tracking performance.

and RA-VM filters, and this result indicates that the RA-VM filter is better in terms of tracking performance. The reason for these findings is as follows. As described in Section 4, the RV-VM filter assumes random velocity and the RA-VM filter assumes random acceleration. Consequently, the RA-VM filter operates to reduce the predicted errors of an accelerating target, and this leads to better tracking performance than in the RV-VM filter. However, there is a trade-off between the tracking and smoothing performance, as indicated in Equations (30) and (38). Hence, the smoothing performance of the RA-VM filter is worse than that in the RV-VM filter. Thus, in practical applications, the RA-VM filter is suitable when better tracking performance is required, whereas the $\mathrm{RV}-\mathrm{VM}$ filter is preferable for better smoothing performance.

\subsection{Application Example}

Finally, we investigate the performances of the proposed VM- $\alpha-\beta$ filters and conventional $\alpha-\beta$ filters in a realistic situation, using numerical simulations of a maneuvering target tracking. For simplicity, we assume one-dimensional tracking. Figure 7 shows the true position and velocity $x_{\mathrm{t}}(t)$ and $v_{\mathrm{t}}(t)$ of the target, where $t=k T$. The sampling interval $T$ is $1 \mathrm{~s}$. The measured position and velocity $x_{0}(t)$ and $v_{0}(t)$ are generated by adding white Gaussian noise to $x_{\mathrm{t}}(t)$ and $v_{\mathrm{t}}(t)$. The variance of the measured position $B_{\mathrm{x}}$ is $1 \mathrm{~m}^{2}$. We investigate $\alpha$ gains of 0.4 and 0.8 and variances of measured velocity $B_{\mathrm{v}}$ of 0.5 and $1 \mathrm{~m}^{2} / \mathrm{s}^{2}$, which correspond to $R_{\mathrm{B}}=0.5$ and 1 . The mean error and mean variance of the position errors are determined for 1000 Monte-Carlo simulations, and these correspond to the tracking and smoothing performance, respectively. The mean error is defined as

$$
e_{\text {mean }}(t)=\frac{1}{1000} \sum_{i=1}^{1000}\left[x_{\mathrm{t}}(t)-x_{\mathrm{p} i}(t)\right]
$$

where $x_{\mathrm{p} i}(t)$ is the predicted position estimated in the $i$-th Monte-Carlo simulation. The mean of the error variance is

$$
\sigma_{\text {mean }}^{2}(t)=\frac{1}{1000} \sum_{i=1}^{1000}\left[e_{\text {mean }}(t)-x_{\mathrm{p} i}(t)\right]^{2}
$$

Figure 8 shows the simulation results for $R_{\mathrm{B}}=0.5$. Figure 8(a) and Figure 8(b) show $\sigma_{\text {mean }}^{2}$ and $e_{\text {mean }}$ for $\alpha$ $=0.4$. In terms of the smoothing performance expressed by $\sigma_{\text {mean }}^{2}$, the conventional filters are better than the VM- $\alpha-\beta$ filters. This is in spite of the fact that $\sigma_{\mathrm{p}}{ }^{2}$ of these filters shown in Figure 2 and Figure 4 are almost the same when $\alpha=0.4$. This is because we have assumed that the covariance of the position and velocity is zero, but this is not completely satisfied in realistic situations. Thus, the variance of errors becomes slightly larger compared with the theoretical value. In contrast, the tracking bias errors $e_{\text {mean }}$ of the VM- $\alpha-\beta$ filters are smaller than those of the conventional filters, as in the theoretical analysis of $e_{\text {fin }}$ shown in Figure 3 and Figure 5. Thus, when the gain $\alpha$ is relatively small and $R_{\mathrm{B}}$ is sufficiently small, the RV-VM and RA-VM filters can improve the tracking performance by using the measured velocity, even in realistic situations. As indicated for $t>700 \mathrm{~s}$, the RA-VM filter gives the best reduction in tracking bias errors for an accelerating target. Figure 8(c) and Figure 8(d) show the results for $\alpha=0.8$. As shown in these figure parts, both the tracking and smoothing performance 


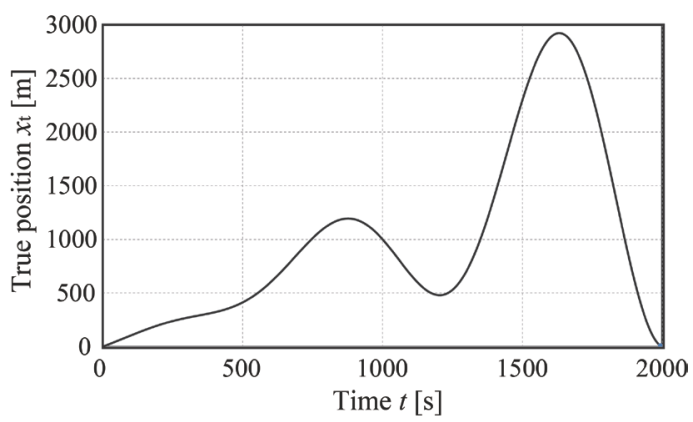

(a)

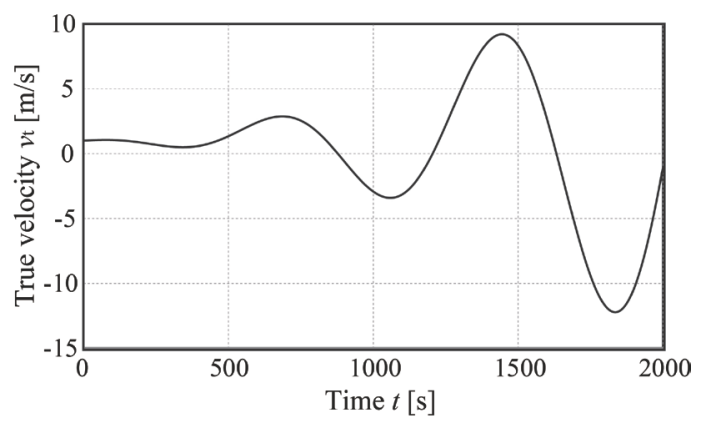

(b)

Figure 7. True position and velocity in numerical simulations. (a) True position $x_{\mathrm{t}}(t)$; (b) True velocity $v_{\mathrm{t}}(t)$.

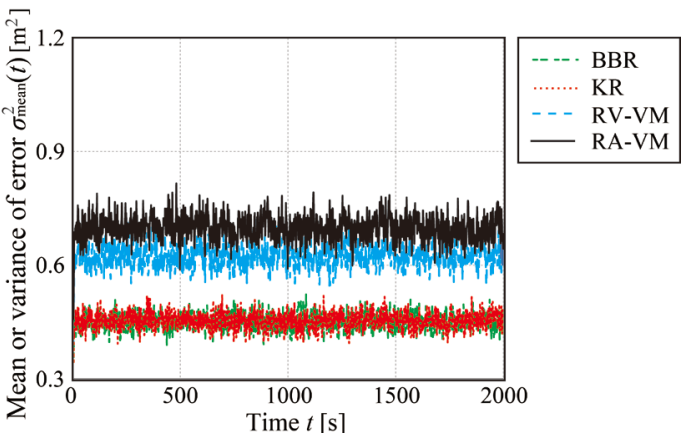

(a)

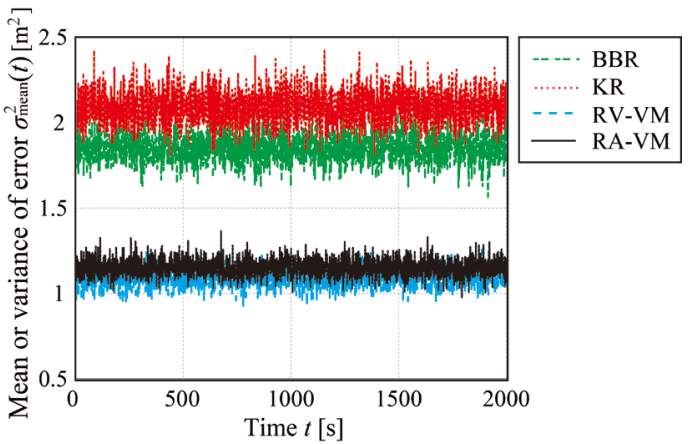

(c)

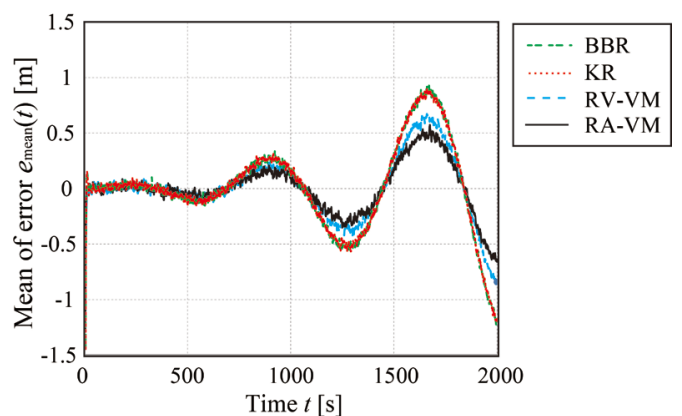

(b)

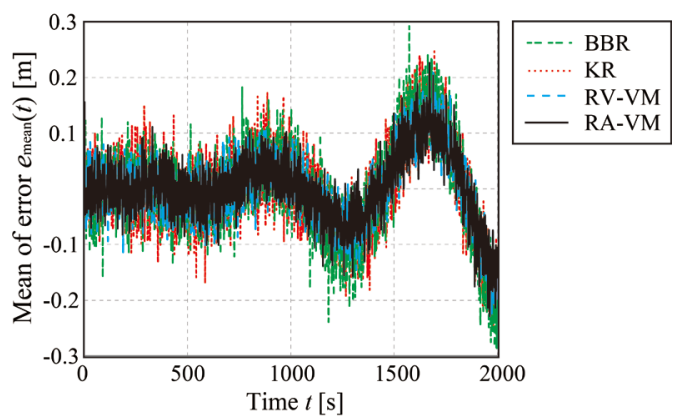

(d)

Figure 8. Numerical simulation results for $R_{\mathrm{B}}=0.5$. (a) Mean of variance of error $(\alpha=0.4)$; (b) Mean of error ( $\alpha=0.4)$; (c) Mean of variance of error $(\alpha=0.8)$; (d) Mean of error $(\alpha=0.8)$.

of the RV-VM and RA-VM filters are better than those of the BBR and KR filters. This implies that $R_{\mathrm{B}}=0.5$ is sufficiently small to allow the filter performance to be improved by using the measured velocity. As indicated in Figure 8(c), the RV-VM filter is the best in terms of smoothing performance. The variance of errors of the RV-VM filter is approximately 1/2 that of the BBR filter in this case. Because the acceleration of the target is not particularly large, the difference in tracking performance is small, as shown in Figure $\mathbf{8}(\mathrm{d})$. However, we can see that the smallest errors are given by the RA-VM filter. Thus, when $\alpha$ is relatively large, the VM- $\alpha-\beta$ filter is effective when the velocity measurement is sufficiently accurate, even in realistic situations.

Figure 9 shows the simulation results for $R_{\mathrm{B}}=1$. Figure 9(a) and Figure 9(b) show the results for $\alpha=0.4$ and these indicate that the smoothing performance of the RV-VM and RA-VM filters is worse than that of the conventional filters, as for $R_{\mathrm{B}}=0.5$. The tracking performance of the RA-VM filter becomes the same as that of the BBR and KR filters. These results suggest that no performance improvement should be expected when the gains are relatively small and the reliability of the velocity sensor cannot be guaranteed. However, Figure 9(c) and Figure 9(d) show the results for $\alpha=0.8$, and these indicate the VM- $\alpha-\beta$ filter can improve the performance 
in this case. As shown in Figure 9(c), the variance of errors of the RV-VM filter is approximately 3/4 of that of the BBR filter. Moreover, the tracking performance of the RA-VM filter is slightly better than that of other filters for $t>1000 \mathrm{~s}$, as shown in Figure 9(d). These results indicate that, even when the reliability of the velocity measurement is the same as that of the position measurement, the derived RV-VM and RA-VM filters realize more accurate tracking than the conventional filters for relatively large filter gains. Moreover, the above discussion indicates that the theoretical calculations and examinations in Sections 5.1-5.3 give almost identical results as the application example of a realistic situation in this subsection. Consequently, the properties derived in this paper will be useful for filter design in actual tracking systems.

\section{Summary and Conclusion}

This paper focused on the general properties of the VM- $\alpha-\beta$ filter. We firstly derived steady-state errors, stability condition, and optimal gains of the VM- $\alpha-\beta$ filter. Table 1 shows a summary of the derived properties of the

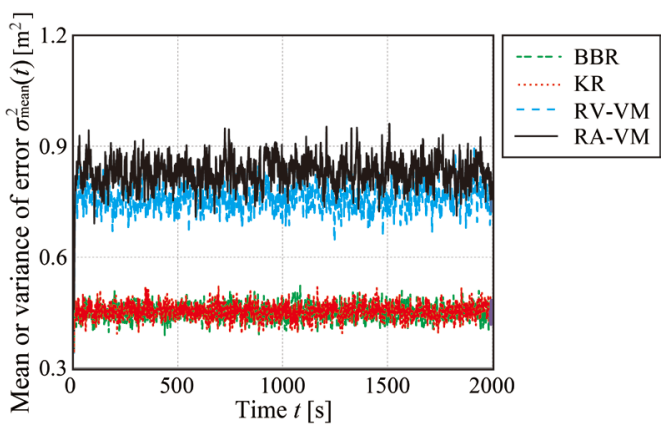

(a)

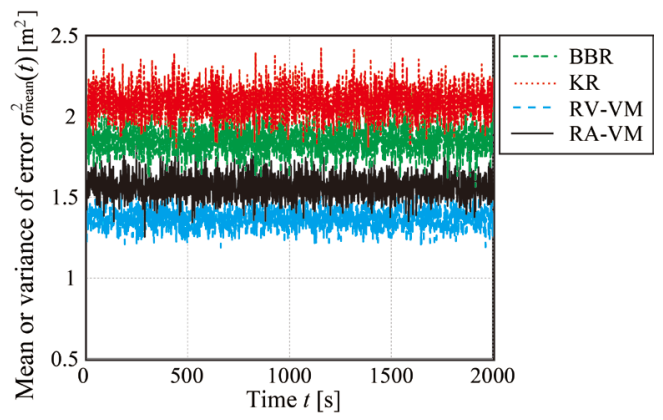

(c)

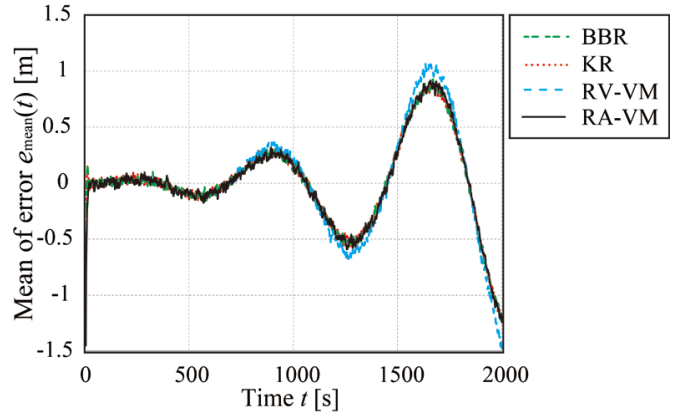

(b)

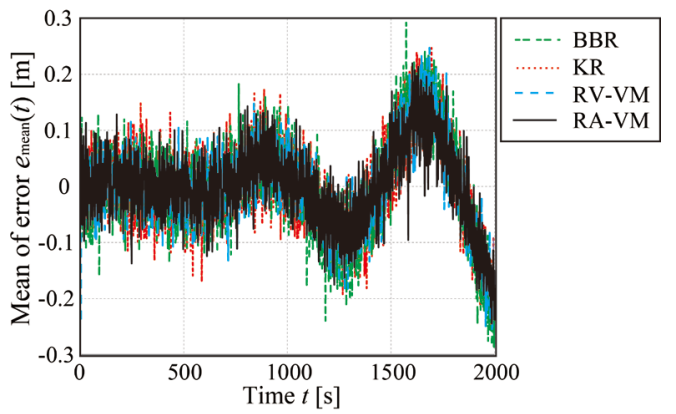

(d)

Figure 9. Numerical simulation results for $R_{\mathrm{B}}=1$. (a) Mean of variance of error $(\alpha=0.4)$; (b) Mean of error $(\alpha=0.4)$; (c) Mean of variance of error $(\alpha=0.8)$; (d) Mean of error $(\alpha=0.8)$.

Table 1. Summary of the properties of the VM- $\alpha-\beta$ filter and conventional $\alpha-\beta$ filter.

\begin{tabular}{|c|c|c|c|c|}
\hline & \multicolumn{2}{|c|}{ VM- $\alpha-\beta$ filter (Equations (8), (10), (11), and (26)) } & \multicolumn{2}{|c|}{ Conventional $\alpha-\beta$ filter (Equations (8)-(11)) } \\
\hline & RV-VM filter & RA-VM filter & BBR filter & KR filter \\
\hline Gain relation & Equation (54) ${ }^{*}$ & Equation (69) ${ }^{*}$ & Equation (16) & Equation (19) \\
\hline Assumed process noise & Equations (14) and (15) & Equations (17) and (18) & Equations (14) and (15) & Equations (17) and (18) \\
\hline Stability condition & Equation (29) ${ }^{*}$ & $\begin{array}{l}\text { Equation (29) and } \\
R_{\mathrm{B}} \neq 0 \text { and } \alpha \neq 1^{*}\end{array}$ & $\begin{array}{c}0<\alpha \text { and } 0<\beta<4-2 \alpha \\
{[6]}\end{array}$ & $\begin{array}{c}0<\alpha \text { and } \alpha \neq 1 \\
\text { and } 0<\beta<4-2 \alpha[6]\end{array}$ \\
\hline Tracking performance & \multicolumn{2}{|c|}{ Equation $(30)^{*}$} & \multicolumn{2}{|c|}{ Equation (13) } \\
\hline Smoothing performance & \multicolumn{2}{|c|}{ Equation (38) ${ }^{*}$} & \multicolumn{2}{|c|}{ Equation (12) } \\
\hline Suitable for & Smoothing* & Tracking* & Smoothing & Tracking \\
\hline $\begin{array}{l}\text { Relatively effective } \\
\text { when the gains are }\end{array}$ & Large $^{*}$ & Large $^{*}$ & Small $^{*}$ & Small $^{*}$ \\
\hline
\end{tabular}

${ }^{*}$ Novel properties clarified in this paper. 
VM- $\alpha-\beta$ filter and conventional $\alpha-\beta$ filters. We then investigated the filter performance of the derived RV-VM and RA-VM filters using numerical calculations based on the derived equations. These calculations confirmed that both the tracking and smoothing performance could be improved using the derived filters when the ratio of position and velocity measurement noise RB is sufficiently small and/or the gains are relatively large. Moreover, numerical simulations showed the effectiveness of the VM- $\alpha-\beta$ filter in a realistic situation. The variance of predicted errors given by the VM- $\alpha-\beta$ filters was approximately $3 / 4$ that of the conventional $\alpha-\beta$ filters, even when the reliabilities of the position and velocity sensors were the same $(\mathrm{RB}=1)$. Moreover, we confirmed the correspondence between our theoretical analysis based on the derived properties and the application results. This implies that the derived properties will be useful for the practical design of tracking systems. However, for simplicity, this paper assumed that the only non-zero elements in the gain and covariance matrices were on the main diagonal. Further analysis without these assumptions is an important future task. Moreover, another possible topic for future work is to analyze properties of tracking filter using other measured parameters such as acceleration.

\section{References}

[1] Ekstrand, B. (2012) Some Aspects on Filter Design for Target Tracking. Journal of Control Science and Engineering, 2012, Article ID: 870890. http://dx.doi.org/10.1155/2012/870890

[2] Kalman, R.E. (1960) A New Approach to Linear Filtering and Prediction Problems. ASME Journal of Basic Engineering, 82, 35-45. http://dx.doi.org/10.1115/1.3662552

[3] Niknejad, H., Takeuchi, A., Mita, S. and McAllester, D. (2012) On-Road Multivehicle Tracking Using Deformable Object Model and Particle Filter with Improved Likelihood Estimation. IEEE Transactions on Intelligent Transportation Systems, 13, 748-758. http://dx.doi.org/10.1109/TITS.2012.2187894

[4] Gross, J., Gu, Y., Gururajan, S. and Seanor, B. (2004) A Comparison of Extended Kalman Filter, Sigma-Point Kalman Filter, and Particle Filter in GPS/INS Sensor Fusion. Proceedings of the IEEE Intelligent Vehicles Symposium, Parma, 14-17 June 2004, 831-835. http://dx.doi.org/10.2514/6.2010-8332

[5] Koteswara, S. (2005) Modified Gain Extended Kalman Filter with Application to Bearings-Only Passive Maneuvering Target Tracking. IEE Proceedings Radar, Sonar and Navigation, 152, 239-244. http://dx.doi.org/10.1049/ip-rsn:20045074

[6] Tenne, D. and Singh, T. (2002) Characterizing Performance of $\alpha-\beta-\gamma$ Filters. IEEE Transactions on Aerospace \& Electronic Systems, 38, 1072-1087. http://dx.doi.org/10.1109/TAES.2002.1039425

[7] Benedict, T. and Bordner, G. (1962) Synthesis of Optimal Set of Radar Track-While-Scan Smoothing Equations. IRE Transactions on Automatic Control, 7, 27-32. http://dx.doi.org/10.1109/TAC.1962.1105477

[8] Bridgewater, A. (1970) Analysis of Second and Third Order Steady State Tracking Filters. Proceedings of AGARD Strategies for Automatic Track Initiation, Ottawa, 9-1-9-11.

[9] Navarro, A. (1977) General Properties of Alpha Beta, and Alpha Beta Gamma Tracking Filters. National Defence Research Organization, TNO.

[10] Kalata, P.R. (1984) The Tracking Index: A Generalized Parameter for at and Target Trackers. IEEE Transactions on Aerospace and Electronic Systems, AES-20, 174-182. http://dx.doi.org/10.1109/TAES.1984.310438

[11] Blackman, S.S. (1986) Multiple Target Tracking with Radar Applications. Artech House, Dedham.

[12] Gray, J. and Murray, W. (1991) The Response of the Transfer Function of an Alpha-Beta Filter to Various Measurement Models. Proceedings of 23rd Southeastern Symposium on System Theory, Columbia, 10-12 March 1991, 389-393. http://dx.doi.org/10.1109/SSST.1991.138587

[13] Hasan, A.H. and Grachev, A.N. (2013) Adaptive $\alpha$ - $\beta$-Filter for Target Tracking Using Real Time Genetic Algorithm. Journal of Electrical and Control Engineering, 3, 32-38.

[14] Wu, C., Chang, C. and Chu, T. (2011) An Implementation of Target Tracking by New GA-Based $\alpha-\beta-\gamma-\delta$ Filter with Its Parameters Optimized by the Taguchi Method Source. International Journal of Organizational Innovation, 4, 148-164.

[15] Mohammed, D., Mokhtar, K., Abdelaziz, O. and Abdelkrim, M. (2009) A New IMM Algorithm Using Fixed Coefficients Filters (fastIMM). International Journal of Electronics and Communications, 64, 1123-1127. http://dx.doi.org/10.1016/j.aeue.2009.11.009

[16] Chernoguz, N. (2007) Adaptive-Gain Kinematic Filters of Orders 2-4. Journal of Computers, 2, 17-25. http://dx.doi.org/10.4304/jcp.2.6.17-25

[17] Baratchi, M., Meratnia, N., Havinga, P.J.M., Skidmore, A.K. and Toxopeus, B.A.G. (2013) Sensing Solutions for Collecting Spatio-Temporal Data for Wildlife Monitoring Applications: A Review. Sensors, 5, 6054-6088. 
http://dx.doi.org/10.3390/s130506054

[18] Valenti, R.G., Dryanovski, I. and Xiao, J.Z. (2013) A Non-Inertial Acceleration Suppressor for Low Cost Inertial Measurement Unit Attitude Estimation. Proceedings of 2013 IEEE International Conference on Robotics and Biomimetics (ROBIO), Shenzhen, 12-14 December 2013, 639-644. http://dx.doi.org/10.1109/ROBIO.2013.6739531

[19] Zheng, C. (2010) Tracking Vehicular Motion-Position Using V2V Communication. Master Thesis, The University of Waterloo.

[20] Ramachandra, K.V., Mohan, B.R. and Geetha, B.R. (1993) A Three-State Kalman Tracker Using Position and Rate Measurements. IEEE Transactions on Aerospace and Electronic Systems, 29, 215-222. http://dx.doi.org/10.1109/7.249127

[21] Jonghyuk, K. and Salah, S. (2005) 6DoF SLAM Aided GNSS/INS Navigation in GNSS Denied and Unknown Environments. Journal of Global Positioning Systems, 4, 120-128. http://dx.doi.org/10.5081/jgps.4.1.120

[22] Gazit, R. (1997) Digital Tracking Filters with High Order Correlated Measurement Noise. IEEE Transactions on Aerospace and Electronic Systems, 33, 171-177. http://dx.doi.org/10.1109/7.570736

[23] Fitzgerald, R.J. (1982) Simple Tracking Filters: Position and Velocity Measurements. IEEE Transactions on Aerospace and Electronic Systems, AES-18, 531-537. http://dx.doi.org/10.1109/TAES.1982.309265

[24] Farina, A. and Pardini, S. (1979) Multiradar Tracking System Using Radial Velocity Measurements. IEEE Transactions on Aerospace and Electronic Systems, AES-15, 555-563. http://dx.doi.org/10.1109/TAES.1979.308740

[25] Gelb, A. (1974) Applied Optimal Estimation. The M.I.T. Press, Cambridge. 